\title{
EFFECTS OF ELECTRONS ON THE SOLAR WIND PROTON TEMPERATURE ANISOTROPY
}

\author{
M. J. MichNO ${ }^{1}$, M. LAZAR ${ }^{1,2}$, P. H. YoON ${ }^{3,4}$, AND R. SCHLICKEISER ${ }^{1,5}$ \\ ${ }^{1}$ Institut für Theoretische Physik, Lehrstuhl IV: Weltraum- und Astrophysik, Ruhr-Universität Bochum, \\ D-44780 Bochum, Germany; mjm@tp4.rub.de, mlazar@tp4.rub.de,rsch@tp4.rub.de \\ 2 Center for Plasma Astrophysics, Celestijnenlaan 200B, B-3001 Leuven, Belgium \\ ${ }^{3}$ Institute for Physical Science and Technology, University of Maryland, College Park, MD 20742, USA; yoonp@umd.edu \\ ${ }^{4}$ School of Space Research, Kyung Hee University, Yongin-Si, Gyeonggi-Do 446-701, Korea \\ ${ }^{5}$ Research Department Plasmas with Complex Interactions, Ruhr-Universität Bochum, D-44780 Bochum, Germany \\ Received 2013 October 24; accepted 2013 December 1; published 2014 January 7
}

\begin{abstract}
Among the kinetic microinstabilities, the firehose instability is one of the most efficient mechanisms to restrict the unlimited increase of temperature anisotropy in the direction of an ambient magnetic field as predicted by adiabatic expansion of collision-poor solar wind. Indeed, the solar wind proton temperature anisotropy detected near $1 \mathrm{AU}$ shows that it is constrained by the marginal firehose condition. Of the two types of firehose instabilities, namely, parallel and oblique, the literature suggests that the solar wind data conform more closely to the marginal oblique firehose condition. In the present work, however, it is shown that the parallel firehose instability threshold is markedly influenced by the presence of anisotropic electrons, such that under some circumstances, the cumulative effects of both electron and proton anisotropies could describe the observation without considering the oblique firehose mode.
\end{abstract}

Key words: instabilities - plasmas - solar wind

Online-only material: color figure

\section{INTRODUCTION}

In the solar wind and the Earth's magnetosheath, the occurrence of anisotropic velocity distributions, i.e., including systems with different temperatures $T_{\|} \neq T_{\perp}$ with respect to the direction of the local magnetic field, is a well-established feature (Kasper et al. 2002; Štverák et al. 2008). This anisotropy can be attributed to the Chew-Goldberg-Low mechanism (Chew et al. 1956) in the solar wind expansion and magnetic focusing, which are counterbalanced by the anisotropy-driven instabilities (Eviatar \& Schulz 1970; Gary et al. 1976). Firehose instabilities are driven by an excess of parallel temperature $A=T_{\perp} / T_{\|}<1$ of plasma particles. The in situ observations confirm that these instabilities could contribute to the isotropization of the solar wind protons (Kasper et al. 2002; Bale et al. 2009; Maruca et al. 2011), electrons (Štverák et al. 2008), and helium ions (Maruca et al. 2012; Bourouaine et al. 2013).

Historically, the firehose instability of the low-frequency (Alfvénic) modes was first discussed in the MHD context (Parker 1958; Vedenov et al. 1961; Davidson \& Völk 1968), and later with kinetic (Vlasov) approaches (Kennel \& Petschek 1966). The instability driven by excessive proton parallel temperature is called the proton firehose instability (PFHI) by many authors (Kennel \& Scarf 1968; Gary \& Feldman 1978; Yoon et al. 1993; Gary et al. 1998). Soon thereafter, a second instability driven by anisotropic electrons was identified (Hollweg \& Völk 1970), which is known as the electron firehose instability (EFHI; Pilipp \& Völk 1971; Gary \& Madland 1985; Gary \& Nishimura 2003; Paesold \& Benz 1999, 2003; Messmer 2002; Camporeale \& Burgess 2008). Due to the kinetic effects of plasma particles both instabilities have finite wave-frequencies and maximum growth at propagation parallel to the magnetic field.

Numerical investigations have also revealed the aperiodic branch of the firehose instability, which is present only at oblique directions. This is called the oblique firehose instability of the protons (Hellinger \& Matsumoto 2000; Hellinger et al. 2006) or the electrons (Li \& Habbal 2000), and seems to dominate over the parallel firehose in the nonlinear evolution. The oblique firehose instability was first discussed by Yoon et al. (1993) in their approximate analytic theory, and later independently rediscovered by Hellinger \& Matsumoto (2000) by means of more accurate but brute-force numerical root finding scheme and on hybrid simulation.

As shown by Hellinger et al. (2006) and Bale et al. (2009), it is a common practice in the literature to display the solar wind data in the phase space of the proton temperature ratio, $T_{\perp} / T_{\|}$, and parallel proton beta, $\beta_{\|}=8 \pi n T_{\|} / B_{0}^{2}$, where $n$ and $B_{0}$ represent the solar wind plasma number density and local magnetic field intensity, respectively. On the basis of such a data analysis technique and linear stability analysis of PFHI, it is generally acknowledged that for parallel temperature anisotropy, $T_{\perp} / T_{\|}<1$, the solar wind plasma is better characterized by the anisotropy lower bound dictated by the oblique firehose marginal instability condition rather than that of the parallel (proton) firehose instability (Hellinger et al. 2006).

The purpose of the present work is to demonstrate that there exists an alternative way to interpret the solar wind data in $\left(T_{\perp} / T_{\|}, \beta_{\|}\right)$space by taking the anisotropic electrons into account, as well as anisotropic protons in the linear stability analysis of the firehose mode. Despite the fact that oblique firehose instability is discussed in the literature, the analytical properties of the oblique mode is poorly understood at present, and thus it will be excluded in this study. Of course, for a general situation, the solar proton anisotropy will be constrained by both the oblique firehose and the cumulative electron-and-ion firehose marginal conditions. The aim of the present paper is to elucidate the effects of the latter condition.

The organization of the present paper is as follows. In Section 2 we introduce kinetic dispersion relation for parallel 

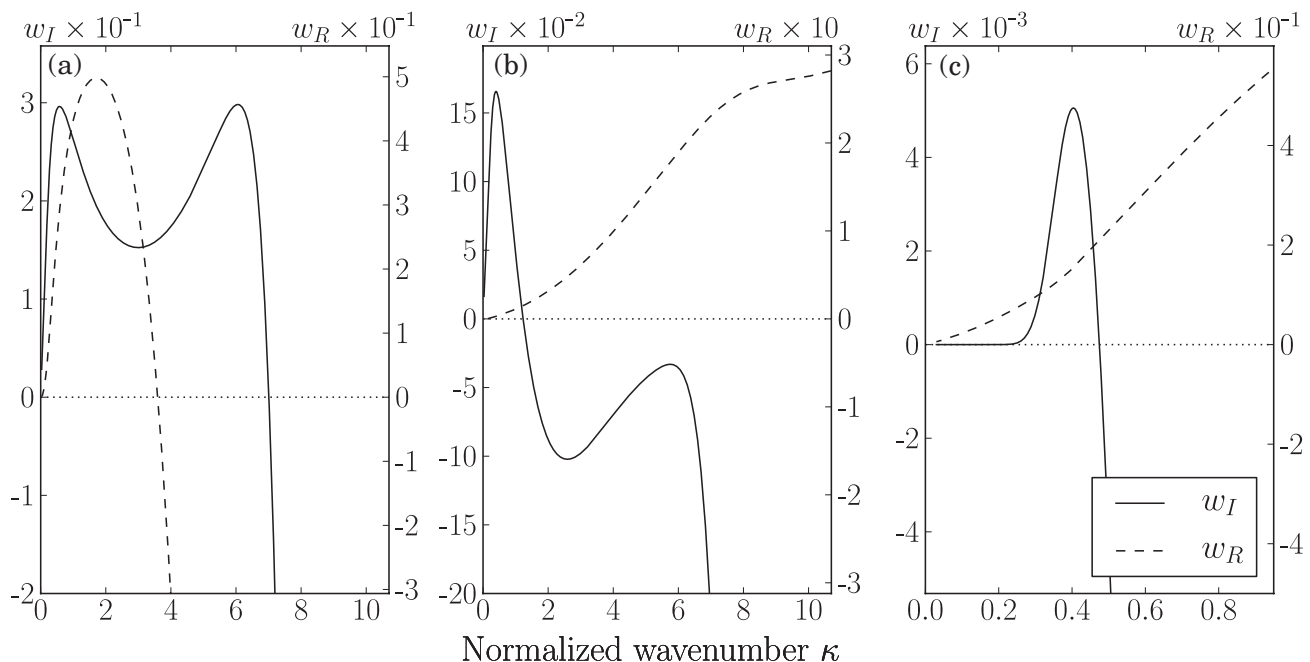

Figure 1. Numerical solutions of Equation (3) generally show growth/damping rates $\left(w_{I}\right)$ with two peaks driven by protons at low wave numbers and by electrons at higher wave numbers (solid lines in left panels). The real frequency $\left(w_{R}\right)$ is displayed with dashed lines. The temperature parameters are (a) $A_{p}=0.4, A_{e}=0.5$, $\beta_{\|, p}=3.0, \beta_{\|, e}=4.0$, (b) $A_{p}=0.4, A_{e}=0.8, \beta_{\|, p}=3.0, \beta_{\|, e}=4.0$, and (c) $A_{p}=0.828, A_{e}=0.7, \beta_{\|, p}=1.5, \beta_{\|, e}=5.6$.

electromagnetic modes in magnetized bi-Maxwellian plasmas. The instability threshold conditions are discussed in Section 3, and comparisons are made with the solar wind data. We show that the cumulative effects of electron and proton anisotropies can lead to a satisfactory explanation of the solar wind proton anisotropy threshold. Our results and conclusions are summarized in the last section.

\section{DISPERSION FORMALISM}

Despite the significant amount of studies on PFHI and EFHI, much of the present understanding stems from simulations using simplified assumptions. Analytical descriptions are in general restrained to the PFHI in the fluid (MHD) limit (Vedenov et al. 1961; Davidson 1984; Hau \& Sonnerup 1993). Kinetic solutions are fluid-like in the sense that they are only valid for small growth rates and wave numbers, and fail to correctly describe the peaks and cutoffs associated with the growth rate. Notable in this sense is an earlier attempt by Pilipp \& Völk (1971) to analyze the EFHI for sufficiently large growth rates.

Among the most striking simplifications associated with the study of EFHI in the literature is the assumption of isotropic protons. Similarly, for studies on the PFHI isotropic electrons are assumed. However, for a general situation, the electrons and protons both contribute to the instability, making it difficult to isolate their independent contributions. The pioneering work of Kennel \& Scarf (1968) demonstrated that anisotropic electrons considerably change the growth rate of the PFHI. More recently, Lazar et al. (2011) discussed the unstable firehose solutions under the cumulative effects of both anisotropic protons and electrons. Our approach is similar but the main difference is that while Lazar et al. (2011) emphasized the role of suprathermal particles on the marginal firehose condition, we shall presently consider the cumulative effects of both electron and proton anisotropies.

We consider a homogeneous, collisionless, and quasi-neutral electron-proton plasma immersed in a constant, ambient magnetic field $\mathbf{B}=B_{0} \mathbf{e}_{z}$. To model the temperature anisotropy, we use bi-Maxwellian distribution functions for both species (Gary et al. 2001; Maksimovic et al. 2005). This is an approximative representation of the real distributions in the solar wind, describing accurately the core population and neglecting the other, more dilute components (suprathermal halo, strahl). However, the success in explaining the bounds of the solar wind temperature anisotropy (Štverák et al. 2008; Bale et al. 2009) motivates this assumption, which may be a helpful step toward a better understanding of kinetic instabilities in the solar wind. The public WIND Solar Wind Experiment (SWE)/Magnetic Field Investigation (MFI) proton moment data used in this study has also been generated with the assumption of bi-Maxwellian protons (Kasper et al. 2002). Plasma modes are small-amplitude perturbations of the steady-state, described by the linearized Maxwell-Vlasov equations. Subject to a Fourier transform, the resulting dispersion relation admits harmonic solutions, i.e., $\propto \exp (i k x-i \omega t)$, which relates complex frequency $\omega=\omega_{R}+i \gamma$ to the real wavenumber $\mathbf{k}$. The imaginary part $\gamma$ is associated with an exponential growth $(\gamma>0)$ or damping $(\gamma<0)$ of the mode. For parallel propagation transverse and longitudinal solutions are decoupled, and transverse electromagnetic modes are described by the textbook dispersion relation (Seough \& Yoon 2012; Yoon \& Seough 2012; Seough et al. 2013)

$$
\begin{aligned}
& 1-\frac{c^{2} k^{2}}{\omega^{2}}+\sum_{a} \frac{\omega_{p, a}^{2}}{\omega^{2}}\left[\frac{T_{\perp, a}}{T_{\|, a}}-1+\left\{\frac{T_{\perp, a}}{T_{\|, a}} \omega\right.\right. \\
&\left.\left. \pm\left(\frac{T_{\perp, a}}{T_{\|, a}}-1\right) \Omega_{a}\right\} \frac{1}{k \alpha_{\|, a}} Z\left(\frac{\omega \pm \Omega_{a}}{k \alpha_{\|, a}}\right)\right]=0,
\end{aligned}
$$

where we sum over each particle species, e.g., $a=e$ for electrons and $a=p$ for protons, and use the following notations: $\omega_{p, a}=\sqrt{4 \pi n_{a} q_{a}^{2} / m_{a}}$ is the plasma frequency, $\Omega_{a}=q_{a} B_{0} /\left(m_{a} c\right)$ is the gyrofrequency, and $c$ is the speed of light. The temperature is assumed anisotropic, and is modeled by a bi-Maxwell velocity distribution function $f_{a}(\mathbf{v}) \propto$ $\exp \left(-v_{\|}^{2} / \alpha_{\|, a}^{2}\right) \exp \left(-v_{\perp}^{2} / \alpha_{\perp, a}^{2}\right)$, where $\alpha_{\|, a}=\sqrt{2 T_{\|, a} / m_{a}}$ and $\alpha_{\perp, a}=\sqrt{2 T_{\perp, a} / m_{a}}$ are parallel and perpendicular thermal velocities, respectively. $Z$ denotes the plasma dispersion function (Fried \& Conte 1961),

$$
Z(x)=\pi^{-1 / 2} \int_{-\infty}^{\infty} d t \frac{\exp \left(-t^{2}\right)}{t-x}, \quad \Im x>0 .
$$

The signs \pm are respectively associated with the circular righthanded $(\mathrm{RH})$ or left-handed $(\mathrm{LH})$ polarization. Because of 
symmetry with respect to the sign of frequency, we only examine the "+"-sign solution, assigning $\omega_{R}>0$ to $\mathrm{RH}$ polarization, and $\omega_{R}<0$ to $\mathrm{LH}$ polarization. For an excess of parallel temperature, i.e., $T_{\|}>T_{\perp}$, the unstable solutions are low-frequency electromagnetic waves (RH fast-magnetosonic/ whistler), and are driven by the firehose instability. In the opposite case of $T_{\|}<T_{\perp}$, growing solutions are the ioncyclotron modes $\left(\omega_{R}<\Omega_{p}\right)$, or the whistler-electron-cyclotron modes at higher frequencies $\left(\Omega_{p}<\omega_{R}<\left|\Omega_{e}\right|\right)$. The first term in Equation (1) arises due to displacement currents and can be neglected if $v_{\text {Alfven }} \ll c$ (e.g., in the solar wind).

In order to simplify the analysis we rewrite the dispersion relation in dimensionless quantities:

$$
\begin{aligned}
0= & -\kappa^{2}+A_{p}-1+\frac{A_{p} w-1+A_{p}}{\kappa \sqrt{\beta_{\|, p}}} Z\left(\frac{w+1}{\kappa \sqrt{\beta_{\|, p}}}\right) \\
& +\mu\left(A_{e}-1\right)+\mu \frac{A_{e} w+\mu\left(1-A_{e}\right)}{\kappa \sqrt{\mu \beta_{\|, e}}} Z\left(\frac{w-\mu}{\kappa \sqrt{\mu \beta_{\|, e}}}\right),
\end{aligned}
$$

where $w=w_{R}+i w_{I}=\omega / \Omega_{p}, \kappa=k c / \omega_{p, p}, \mu=m_{p} / m_{e}$ is the proton to electron mass ratio, $A_{a}=T_{\perp, a} / T_{\|, a}$ is the temperature anisotropy, and $\beta_{\|, a}=8 \pi n_{a} T_{\|, a} / B_{0}^{2}$ is the parallel plasma beta. The unstable wave solution, given by the complex frequency $w$, depends on the real wave-number $\kappa$, and four additional plasma parameters $A_{p}, A_{e}, \beta_{\|, p}$, and $\beta_{\|, e}$.

Figure 1 displays three representative cases of the combined EFHI and PFHI. For the first set of parameters in Figure 1(a), namely $A_{p}=0.4, A_{e}=0.5, \beta_{\|, p}=3.0$, and $\beta_{\|, e}=4.0$, the unstable solutions display two distinct peaks for the growth rate, and the real frequency changes sign in-between. The first peak (at lower $\kappa$ ) corresponds to a $\mathrm{RH}$ mode driven unstable by the PFHI, and the second peak (larger $\kappa$ ) is the LH mode driven by EFHI. For higher $\kappa$, there is a sharp cutoff and the mode is damped beyond the maximum $\kappa$. In Figure 1(b), for which all the input parameters are the same except that the electrons have a sufficiently low anisotropy $A_{e}=0.8$, which close to unity, the instability is dominated by the proton (low $\kappa$ ) firehose effect, and the second peak (if it exists) is negative (damping). Note that compared to the first panel, the maximum growth rate of the PFHI is decreased by almost a factor two due to the different electron anisotropy. Finally, in Figure 1(c), we consider conditions close to the threshold $\left(w_{I, M} \rightarrow 0\right)$ of the PFHI. As such, we consider $A_{p}=0.828$, which is close to unity, proton beta $\beta_{\|, p}=1.5$, but more anisotropic electrons $A_{e}=0.7$ and high electron parallel beta $\beta_{\|, e}=5.6$. In this case, the proton firehose peak is very small $w_{I, M} \approx 10^{-3}$ (indiscernible visually), and is shifted to lower values of $\kappa<1$. The firehose instability for Figure 1(c) is entirely owing to the electron anisotropy. Only the third case is relevant for explaining the proton isotropization, as low growth rates of the order $w_{I} \sim 10^{-3}$ are sufficient to explain the limits on the data distribution (Bale et al. 2009).

\section{PFHI VERSUS EFHI: THRESHOLD CONDITIONS}

In order to discuss the combined PFHI and EFHI marginal stability condition we numerically calculate the instability conditions by repeatedly solving for marginally stable roots over a range of input parameters. The anisotropy thresholds are obtained by solving the exact dispersion relation (3) for small values of $w_{I}^{M}\left(=10^{-1}, 10^{-2}, 10^{-3}\right)$ close to the marginal stability $\left(w_{I}^{M} \rightarrow 0\right)$. The growth rate dependence on both

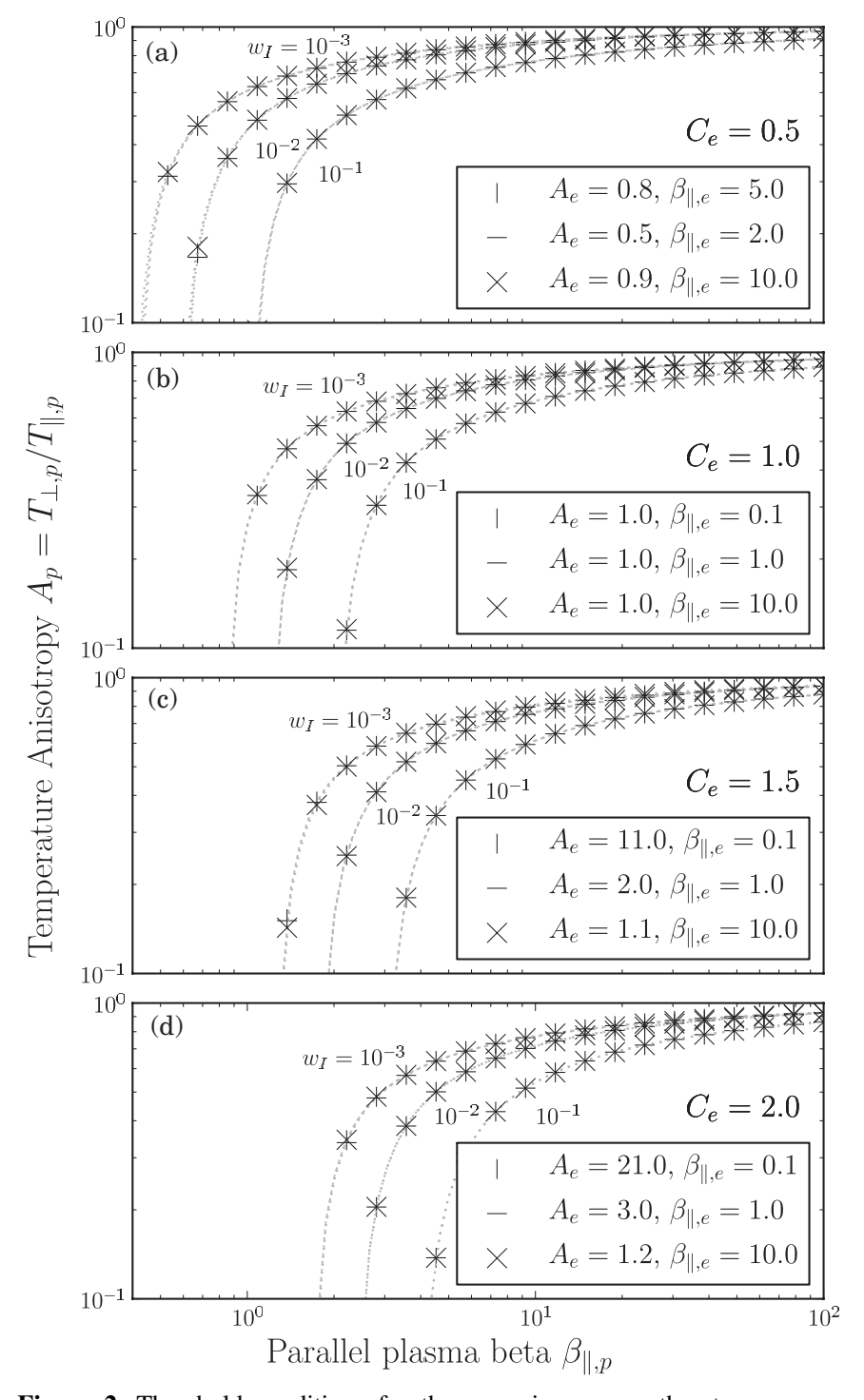

Figure 2. Threshold conditions for three maximum growth rates $w_{I, M}=$ $10^{-3}, 10^{-2}, 10^{-1}$ of the PFHI and different parameters $A_{e}$ and $\beta_{\|, e}$ illustrate the dependence of only one combined parameter $C_{e}=1-\left(1-A_{e}\right) \beta_{\|, e} / 2$.

particle species is investigated in turn. We determine the anisotropy thresholds $A_{p}\left(\beta_{\|, p}\right)$ as a function of plasma beta parameter for each given value of the growth rate $w_{I}=10^{-1}$, $10^{-2}, 10^{-3}$, and fit the results with the inverse correlation law (Hellinger et al. 2006),

$$
A_{p}=1+\frac{a}{\left(\beta_{\|, p}-\beta_{0}\right)^{b}} .
$$

The more commonly used fitting formula (Gary \& Wang 1996) that omits the fitting parameter $\beta_{0}(\equiv 0)$, makes it impossible to account for the shift of the thresholds to higher $\beta_{\|, p}$ values. We thus use Equation (4) as the empirical functional form, but in contrast to previous studies, we extend the analysis to include anisotropic electrons for the parallel firehose mode.

In general, the impact of anisotropic electrons enters the dispersion relation via $A_{e}=T_{\perp, e} / T_{\|, e}$ and $\beta_{\|, e}$, as Equation (3) evidently shows. However, we discovered a remarkable property associated with the parallel firehose instability which simplifies the problem. That is, we found that the proton firehose growth rate dependence on the electron quantities is controlled only by 


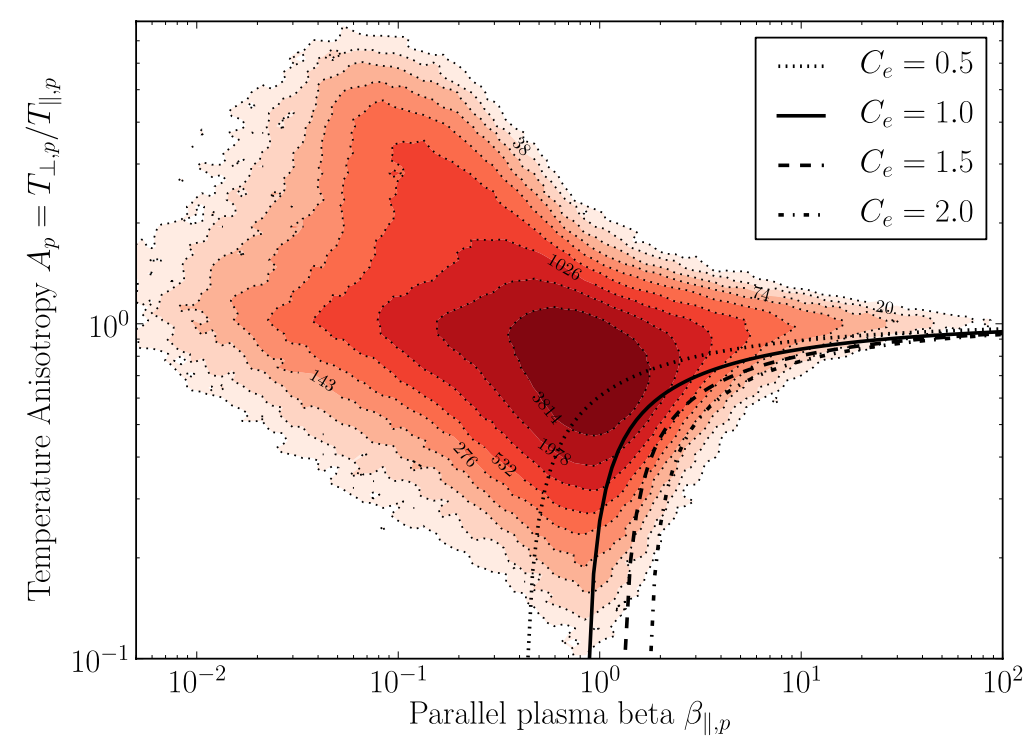

Figure 3. Threshold conditions for $w_{I, M}=10^{-3}$ and four different values of $C_{e}=0.5,1.0,1.5,2.0$ are compared with the proton data at $1 \mathrm{AU}$ in the solar wind. Labels indicate the isocontours with the same number of counts.

(A color version of this figure is available in the online journal.)

a single parameter $C_{e}$ defined by

$$
C_{e} \equiv 1-\beta_{\|, e}\left(1-A_{e}\right) / 2,
$$

instead of $A_{e}$ and $\beta_{\|, e}$ separately. To prove this finding, we consider four values $C_{e}=0.5,1.0,1.5$. 2.0, and for each of them, we chose three different combinations for $A_{e}$ and $\beta_{\|, e}$. The numerical results illustrated in Figure 2 confirm our finding. Specifically, in panel (a), we consider three different cases of electron input parameters, $\left(A_{e}, \beta_{\|, e}\right)=(0.8,5.0),(0.5,2.0)$, and $(0.9,10.0)$. Note that all three cases yield the same value of the combined parameter $C_{e}=0.5$. As Figure 2(a) shows, all three cases yield practically indistinguishable results. Three different combinations of $A_{e}$ and $\beta_{\|, e}$ are marked by short vertical dashes, horizontal dashes, and X's, but the roots fall on top of each other. Similarly in panel (b), we considered the case of $C_{e}=1.0$, and three different combinations of $\left(A_{e}, \beta_{\|, e}\right)=(1.0,0.1),(1.0,1.0)$, and $(1.0,10.0)$, that yield the value of $C_{e}=1.0$. In this case, since the electrons are isotropic, the combined parameter $C_{e}$ is independent of $\beta_{\|, e}$. In panel (c) we continue with the case of $C_{e}=1.5$. In this case, we considered three different combinations of $\left(A_{e}, \beta_{\|, e}\right)=(11.0,0.1),(2.0,1.0)$, and $(1.1,10.0)$. Again, we see that all the roots are identical. Finally, panel (d) shows the case of $C_{e}=2.0$ with $\left(A_{e}, \beta_{\|, e}\right)=(21.0,0.1),(3.0,1.0)$, and $(1.2,10.0)$. We have considered other cases and combinations, and have confirmed our finding. We thus conclude that the four different cases of parameter $C_{e}$ combined with three different combinations of $\left(A_{e}, \beta_{\|, e}\right)$, producing the same value of $C_{e}$ is sufficient to prove that the effects of electrons on the parallel firehose instability is indeed controlled by a single parameter $C_{e}=1-\beta_{\|, e}\left(1-A_{e}\right) / 2$. A complete technical argument on the form of the parameter $C_{e}$ is presented in a forthcoming paper (M. J. Michno et al. 2014, in preparation).

Note that the growth rate increases as $C_{e}$ decreases. The marginal contour lines shift to the left-top corner. This implies that the empirical marginal stability curve given by the basic form of Equation (4) will be different for different values of $C_{e}$, and we fit the threshold conditions for each $C_{e}$ accordingly. The empirically determined fitting parameters are given in Table 1. For isotropic electrons $\left(C_{e}=1.0\right)$, we recover a result virtually
Table 1

Fitting Parameters for Equation (4) Empirically Deduced on the Basis of Constant Growth Rates $w_{I, M}=10^{-3}$ Associated with the PFHI

\begin{tabular}{cccc}
\hline \hline$C_{e}$ & $a$ & $b$ & $\beta_{0}$ \\
\hline 0.5 & -0.327 & 0.485 & 0.314 \\
1.0 & -0.453 & 0.467 & 0.652 \\
1.5 & -0.545 & 0.464 & 0.990 \\
2.0 & -0.638 & 0.479 & 1.287 \\
\hline
\end{tabular}

identical to the fit of Hellinger et al. (2006) for the parallel proton firehose case, especially if one compares them in one plot (not shown).

The basic physical mechanism behind this electron anisotropy effect has already been identified by Kennel \& Scarf (1968). The PFHI is non-resonant with the electrons, but the electron anisotropy changes the phase velocity of the mode, which in turn changes the proton cyclotron resonance condition. For isotropic electrons, the protons are weakly resonant, $\xi_{p}=$ $\left|w_{R}+1 / \kappa \sqrt{\beta_{\|, p}}\right| \gtrsim 1$, but changing the electron anisotropy modifies the position of the proton resonance with respect to the peak of the distribution. In particular, increasing $C_{e}$ increases the phase velocity and, thus, the resonance term $\xi_{p}$. The protons become less resonant, leading to lower growth rates and moving the thresholds to the right. A decrease of $C_{e}$ moves $\xi_{p}$ closer to one, so that more protons are resonant and the growth rate increases. A detailed analysis will be presented in a forthcoming paper (M. J. Michno et al. 2014, in peparation).

We may now apply the results we have obtained thus far to explain the limits observed for the temperature anisotropy in the solar wind. The wave-particle scattering by the enhanced fluctuations of growing modes is expected to reduce the anisotropy of plasma particles, and maintain the distributions at, or near the instability threshold. We calculate parameters of the solar wind, namely the temperature anisotropy and the parallel plasma beta, using data from WIND SWE and MFI instruments (through the SPDF CDAWeb service), for the proton velocity distribution and magnetic field, respectively (Ogilvie et al. 1995; Lepping et al. 1995; Bale et al. 2009). The results, counted by the isolines in the parameter space $A_{p}$ versus $\beta_{\|, p}$, are displayed in Figure 3. Each 
bin corresponds to a logarithmically spaced $100 \times 100$ grid in the intervals $10^{-1} \leqslant A_{p} \leqslant 10^{1}$ and $10^{-3} \leqslant \beta_{\|, p} \leqslant 10^{2}$. We discard data with errors larger than $10 \%$ in the thermal speed, and where the spacecraft was too close to the Earth's bow shock. ${ }^{6}$ The instability threshold contours at $w_{I}=10^{-3}$ are also shown for different electron parameters $C_{e}=0.5,1.0,1.5,2.0$. Thresholds are fitted to Equation (4), and fitting parameters are given in Table 1. The isotropic electrons $\left(C_{e}=1.0\right)$ corresponds to that of Hellinger et al. (2006) for the parallel proton firehose marginal stability. As one can see from Figure 3, the parallel firehose threshold apparently does not match up perfectly with the observation. Hellinger et al. (2006) thus conclude that the oblique firehose marginal stability curve better fits the data. However, as Figure 3 shows, the instability thresholds calculated for anisotropic electrons with $A_{e}>1\left(C_{e}>1\right)$, approach the limits of the observed data as $C_{e}$ increases. However, measurements indicate that the core electrons are tightly confined around $A_{e}=\beta_{\|, e}=1$ and show rather an anisotropy in parallel direction, $A_{e} \lesssim 1$ (Štverák et al. 2008). As the needed perpendicular anisotropies $A_{e}>1$ are only measured for a small part of the data distribution, the described effect is probably relevant only for part of the observations.

From this finding, we may conclude that the solar wind proton anisotropy boundary for $A_{p}<1$ may be alternatively explained as being constrained by the parallel firehose instability with anisotropic electrons, or as Hellinger et al. (2006) suggest, it may be understood as the dominant oblique firehose mode dictating the anisotropy boundary.

\section{CONCLUSIONS}

The theory of the firehose instability is in general reduced either to the PFHI, keeping the electron distribution isotropic, or to the EFHI, taking protons as isotropic. In the present paper we have refined the analysis of this instability, assuming both species are anisotropic. The main ingredient of this investigation is the interplay of the EFHI and PFHI, both of which develop concurrently for such realistic models.

Under the cumulative effect of both the anisotropic protons and electrons, the exact firehose solutions can exhibit two distinct peaks for the growth rate, and the real frequency changes sign in between. The first peak (at lower $\kappa$ ) describes the $\mathrm{RH}$ mode driven unstable by the PFHI, and the second peak (larger $\kappa$ ) is the LH mode driven by the EFHI. In the range of the second peak growing modes are inhibited and become damped if the electron anisotropy is not high enough.

We have also examined numerically the mutual influence of the anisotropic protons and electrons on their instability thresholds. In doing so, we have uncovered a useful property associated with PFHI in that the instability thresholds and growth rates only depend on a single combined electron parameter $C_{e}=1+\left(A_{e}-1\right) \beta_{\|, e} / 2$ ( $C_{e}=1$ meaning isotropic electrons). It is also found that lowering $C_{e}$ increases the growth rate, while increasing $C_{e}$ has the opposite effect. This finding was then applied to the solar wind proton temperature anisotropy limit, where previous discussion by Hellinger et al. (2006) suggests that the lower boundary in the case of $A_{p}=T_{\perp, p} / T_{\|, p}<1$ conforms with the oblique firehose marginal condition. However, according to our result, the presence of anisotropic electrons could provide an alternative explanation for the observed property in the context of the parallel firehose instability alone. That

\footnotetext{
6 We require $x>-0.024(y+z)^{2}+20$, where $(x, y, z)$ is the position of the spacecraft in GSE coordinates (Hapgood 1992) in units of Earth radii.
}

is, when $C_{e}>1$, the threshold of the PFHI improves toward the observed anisotropy limit, so that an improved fit with the observed temperature anisotropy limits in the solar wind can be achieved.

We thank the anonymous referee for his constructive comments, which helped to improve the presentation of our paper. The authors acknowledge the use of WIND SWE (Ogilvie et al. 1995) ion data, and WIND MFI (Lepping et al. 1995) magnetic field data from the SPDF CDAWeb service: http://cdaweb.gsfc.nasa.gov/. M.J.M., M.L., and R.S. acknowledge support from the Ruhr-Universität Bochum, and the Deutsche Forschungsgemeinschaft (DFG), grants Schl 201/211 and Sh 21/3-2. M.L. acknowledges financial support from the EU Commission and Research Foundation Flanders (FWO) as FWO Pegasus Marie Curie Fellow (grant 1.2.070.13). P.H.Y. acknowledges support from NSF under grant AGS1242331 to the University of Maryland. The research at Kyung Hee University was supported by the BK21-Plus grant through the National Research Foundation (NRF) funded by the ministry of Education of Korea.

\section{REFERENCES}

Bale, S. D., Kasper, J. C., Howes, G. G., et al. 2009, PhRvL, 103, 211101 Bourouaine, S., Verscharen, D., Chandran, B. D. G., et al. 2013, ApJL, 777, L3

Camporeale, E., \& Burgess, D. 2008, JGR, 113, A07107

Chew, G. F., Goldberger, M. L., \& Low, F. E. 1956, RSPSA, 236, 112

Davidson, R. C. 1984, in Basic Plasma Physics (Vol. I \& II), ed. A. A. Galeev \& R. N. Sudan (Amsterdam: North-Holland), 519

Davidson, R. C., \& Völk, H. J. 1968, PhFl, 11, 2259

Eviatar, A., \& Schulz, M. 1970, P\&SS, 18, 321

Fried, B. D., \& Conte, S. D. 1961, The Plasma Dispersion Function (New York: Academic)

Gary, S. P., \& Feldman, W. C. 1978, PhFl, 21, 72

Gary, S. P., Li, H., O’Rourke, S., \& Winske, D. 1998, JGR, 103, 14567

Gary, S. P., \& Madland, C. D. 1985, JGR, 90, 7607

Gary, S. P., Montgomery, M. D., Feldman, W. C., \& Forslund, D. W. 1976, JGR, 81,1241

Gary, S. P., \& Nishimura, K. 2003, PhP1, 10, 3571

Gary, S. P., Skoug, R. M., Steinberg, J. T., \& Smith, C. W. 2001, GeoRL, 28,2759

Gary, S. P., \& Wang, J. 1996, JGR, 101, 10749

Hapgood, M. A. 1992, P\&SS, 40, 711

Hau, L.-N., \& Sonnerup, B. U. Ö. 1993, GeoRL, 20, 1763

Hellinger, P., \& Matsumoto, H. 2000, JGR, 105, 10519

Hellinger, P., Trávníček, P., Kasper, J. C., \& Lazarus, A. J. 2006, GeoRL, 33,9101

Hollweg, J. V., \& Völk, H. J. 1970, JGR, 75, 5297

Kasper, J. C., Lazarus, A. J., \& Gary, S. P. 2002, GeoRL, 29, 20

Kennel, C. F., \& Petschek, H. E. 1966, JGR, 71, 1

Kennel, C. F., \& Scarf, F. L. 1968, JGR, 73, 6149

Lazar, M., Poedts, S., \& Schlickeiser, R. 2011, A\&A, 534, A116

Lepping, R. P., Acũna, M. H., Burlaga, L. F., et al. 1995, SSRv, 71, 207

Li, X., \& Habbal, S. R. 2000, JGR, 105, 27377

Maksimovic, M., Zouganelis, I., Chaufray, J.-Y., et al. 2005, JGR, 110, A09104

Maruca, B. A., Kasper, J. C., \& Bale, S. D. 2011, PhRvL, 107, 201101

Maruca, B. A., Kasper, J. C., \& Gary, S. P. 2012, ApJ, 748, 137

Messmer, P. 2002, A\&A, 382, 301

Ogilvie, K. W., Chornay, D. J., Fritzenreiter, R. J., et al. 1995, SSRv, 71, 55

Paesold, G., \& Benz, A. O. 1999, A\&A, 351, 741

Paesold, G., \& Benz, A. O. 2003, A\&A, 401, 711

Parker, E. N. 1958, PhRv, 109, 1874

Pilipp, W., \& Völk, H. J. 1971, JPIPh, 6, 1

Seough, J., \& Yoon, P. H. 2012, JGR, 117, A08101

Seough, J., Yoon, P. H., Kim, K.-H., \& Lee, D. H. 2013, PhRvL, 110, 071103

Štverák, Š., Trávníček, P., Maksimovic, M., et al. 2008, JGRA, 113, 3103

Vedenov, A. A., Velikhov, E. P., \& Sagdeev, R. Z. 1961, SvPhU, 4, 332

Yoon, P. H., \& Seough, J. 2012, JGR, 117, A08102

Yoon, P. H., Wu, C., \& de Assis, A. S. 1993, PhFlB, 5, 1971 